\title{
Challenges in neurodegeneration research
}

\section{Wendy Noble ${ }^{1 *}$ and Mark P. Burns ${ }^{2 * *}$}

1 King's College London, London, UK

2 Georgetown University Medical Center, Washington, DC, USA

Correspondence: *wendy.noble@kcl.ac.uk; **mpb37@georgetown.edu

The prevalence of neurodegenerative diseases, particularly those that affect the aged, are predicted to increase rapidly in the coming decades. The World Health Organization forecasts that dementia cases will increase by almost $50 \%$ between 2005 and 2030. Supporting data suggests that the predicted number of cases of Alzheimer's disease will quadruple by 2050. It is estimated that interventions that can delay disease onset or progression by just 1 year would reduce the number of predicted cases of Alzheimer's disease by 9.2 million. Unfortunately, current therapies for most neurodegenerative diseases are symptomatic, and few, if any, disease-modifying strategies are available. However, over the last few decades there have been considerable advances in understanding the triggers of neurodegenerative disease and we remain confident that in the coming decade, new disease modifying strategies will be discovered and proven to be effective. Nevertheless, several challenges remain in the field that we must overcome to allow significant progress.

To fully elucidate mechanisms of disease, it is essential that good model systems are available. While cell culture and computer modelling have pivotal roles in understanding pathways and interactions, nothing is as powerful as an accurate animal model. Many transgenic animals, particularly mice, have been created to model most major neurodegenerative disorders. While these models have undoubtedly been of great benefit, the extent to which they recapitulate the main biochemical and behavioural phenotypes of disease varies greatly. Perhaps nowhere is this more obvious than in Alzheimer's disease research. If you follow the amyloid cascade hypothesis, as many do, then an increase in $A \beta$ production/deposition should evoke several signalling cascades resulting in abnormal tau processing and neuronal death. Yet, the majority of mice genetically altered to over-produce $A \beta$ do not show significant tau pathology, and have relatively little neurodegeneration. Is this simply because mice do not live long enough to develop tau pathology and the associated neuronal death? Or are we simply missing something? A similar problem is true for Parkinson's disease models where the genetic manipulation of several known disease-associated genes, including DJ-1, parkin and $\alpha$-synuclein, often fails to reproduce the selective vulnerability of dopaminergic neurons in the nigrostriatal pathway. With increasing use of more advanced transgenic technology, particularly cell- and tissue-type expression and knock-out of genes with cre-lox technology, we remain hopeful that models that more faithfully recapitulate human disease will be developed.

Another important issue in the field of neurodegeneration research is that we need to fully understand the involvement of protein aggregation in disease progression. Aggregation is a complex process in most neurodegenerative diseases, involving phosphorylation, conformational changes, protein truncation by proteases, polymerisation, and several other modifications. Aggregates are common to most neurodegenerative disorders, presenting as neurofibrillary tangles and senile plaques in Alzheimer's disease, Lewy bodies containing $\alpha$-synuclein in Parkinson's disease, ubiquitinated protein aggregates in motor neuron disease, and accumulations of truncated huntingtin protein in Huntington's disease. Despite years of intensive research, it remains unclear whether protein aggregates exert toxic properties, or if their formation is a protective response by neurons as they attempt to minimise the influence of excess abnormally processed proteins. Several studies in models of Alzheimer's and amyotrophic lateral sclerosis have suggested that aggregate formation can be dissociated from neuronal death, and that early, pre-aggregate forms of proteins may be more neurotoxic than aggregated inclusions. Indeed, soluble monomeric and oligomeric protein species are believed to be more closely associated with neurodegeneration in several diseases. Determining which protein species have inherent toxicity is especially important when considering the large number of therapeutic strategies aimed at reducing aggregate load that are currently being developed. It is entirely possible that dis-aggregating potentially inert disease-associated proteins may increase the amount of toxic pre-aggregated soluble oligomers, thereby worsening rather than preventing disease progression. Therefore, it is vital that we further our understanding of the various abnormal forms of proteins that are generated during the development of disease, to ensure that appropriate therapeutic avenues are followed.

The spread of pathology in neurodegenerative diseases has recently been proposed to occur by prion-like transmission. Indeed, $\mathrm{A} \beta$, tau and $\alpha$-synuclein are reported to share key biophysical and biochemical characteristics with prions. For example, 'seeding' has been reported in several neurodegenerative diseases, where truncated tau species and mutant $\alpha$-synuclein apparently act as a nucleation centre for the aggregation of their full-length or wild-type counterparts. Furthermore, in typical prion diseases, it is believed that the conformation of prion proteins is largely responsible for the different disease phenotypes observed. Perhaps this is also true in fronto-temporal dementias associated with tau mutations on chromosome-17, for which heterogenous phenotypes have been reported. It is possible that further exploration of the prion-like properties of disease-associated proteins may elucidate the mechanisms by which neurodegenerative disease pathology spreads. This is still a poorly understood aspect of this field of research, which is perhaps surprising since selective neuronal vulnerability, and the distribution of pathology during disease progression, has been widely studied and reported for most common neurodegenerative diseases. 
From a clinical perspective, it is clear that more reliable and specific biomarkers of disease are needed. These would allow the rapid diagnosis of neurodegenerative conditions while they are most amenable to treatment, and would permit the monitoring of disease progression, particularly important when assessing the efficacy of new treatments. However, this has proven to be problematic. Most neurodegenerative diseases are complex, involving several proteins, often with accompanying neuroinflammation, and with varying ages of onset and speed of progression. In addition, there is often overlap between the types of pathology observed in distinct diseases. Furthermore, it is relatively common for two or more neurodegenerative diseases to co-occur. Proteomic analysis is increasingly used as an unbiased approach to identify disease markers. Such screening, in combination with neuroimaging and additional diagnostic testing may enable the future development of sensitive and specific biomarkers of neurodegenerative diseases. It will also be important for such studies to follow patients until autopsy so that their findings can be fully validated using postmortem tissue.

Another important aspect when considering treatment is genetic profile. It has become clear in recent years: that a persons genetic profile will alter how they respond to drug treatments. This was notably recorded in recent Alzheimer's disease trials where persons carrying the apoE4 allele did not respond to therapeutic intervention.
Personalized medicine is a long term goal of the medical field and it is becoming increasingly obvious that that genetic background and environmental influences will now have to be taken into consideration when designing new clinical trials. Indeed, investigations of new treatments in pre-clinical laboratory studies should perhaps also be designed to include several background strains of mice since this would provide valuable insight into any potential influences of genetic variability. Another important aspect of clinical research that requires further research is the effectiveness of combination therapies. For example, there appears to be a synergistic relationship between tau and $A \beta$ in Alzheimer's disease, so perhaps a treatment that reduces amyloid load should be used together with a tau-directed approach, and/ or anti-inflammatory agents, for maximum efficacy.

Finally, stem cell therapies have shown some promise for the treatment of neurodegenerative diseases. The central nervous system, unlike many other tissues, has a very limited capacity for self-repair, although there are populations of endogenous neural stem cells in specific brain regions. Perhaps the greatest recent advance in this area is the use of induced pluripotent stem cells (iPSCs). The discovery that fibroblasts can be re-programmed to pluripotency and then differentiated into specific neuronal subtypes has wide-spread implications for the field. Obtaining fibroblasts from skin biopsies of patients with neurodegenerative diseases will allow the study of patient-specific disease mechanisms and progression, and may in the future lead to individually tailored therapeutic interventions. Although there are some technical challenges yet to be overcome in this area of research, it seems clear that the manipulation of iPSCs may represent a powerful new strategy for the treatment of neurodegenerative diseases.

In summary, the field of neurodegenerative disease research has made enormous strides in recent decades. However, the challenge for the future will be the ability to utilise our new found knowledge to develop better tools and novel therapeutic strategies. Importantly, we need better methods to identify people who are at high risk of developing neurodegenerative disease through the use of sensitive and specific biomarkers. Equally, we require more effective therapies that can reduce neuronal loss and prevent disease progression, perhaps by taming stem cells to regenerate damaged central nervous systems, by immunization, or through more conventional pharmacological approaches.

Received: 17 February 2010; accepted: 18 February 2010; published online: 10 March 2010.

Citation: Noble W and Burns MP (2010) Challenges in neurodegeneration research. Front. Psychiatry 1:7. doi: 10.3389/fpsyt.2010.00007

This article was submitted to Frontiers in Neurodegeneration, a specialty of Frontiers in Psychiatry

Copyright $(\odot 2010$ Noble and Burns. This is an open-access article subject to an exclusive license agreement between the authors and the Frontiers Research Foundation, which permits unrestricted use, distribution, and reproduction in any medium, provided the original authors and source are credited. 